\title{
ALGORITHM FOR PRIORITY CHARGING IN A DC MICRO-GRID
}

\author{
U Sowmmiya, Aditya Prasad Chakrabartty and Chaitanya Tyagi \\ Department of Electrical and Electronics Engineering, \\ SRM Institute of Science and Technology, Chennai, Tamilnadu, India
}

\begin{abstract}
With the increasing electric vehicles (EVs), the power grid will be impacted for charging the EVs. In order to manage EVs charging, there is a need for an intelligent charging strategy that supports EVs charging while preventing the power grid from overloading. In this paper, an algorithm is proposed for optimal charging of the batteries based on priority. The priority determination rules are calculated according to source voltage, current state-of-charge (SOC), battery capacity and battery current. The proposed algorithm considers real-time price (RTP) in order to minimize charging cost without increasing peak load. Intelligent charging strategies are needed in order to minimize electric vehicles charging cost without violating the operational constraints of the electric power grid with an extra burden. This work aims to design an optimal scheduling algorithm for managing electric vehicles charging using droop control.
\end{abstract}

Key words: Battery, EV charging, Energy management, Priority determination, SoC.

Cite this Article: U Sowmmiya, Aditya Prasad Chakrabartty and Chaitanya Tyagi, Algorithm for Priority Charging in a DC Micro-Grid. International Journal of Electrical Engineering and Technology, 11(4), 2020, pp. 300-308.

https://iaeme.com/Home/issue/IJEET?Volume $=11 \&$ Issue $=4$

\section{INTRODUCTION}

Electrical Vehicles having advantage of using greener energy rather than fossil fuels so the demand of electrical vehicles are to be increased in the world. As the number of EVs is continuously growing exponentially, a lot of energy will be required to support the grid integration of EVs. Therefore, charging many EVs may affect the power grid stability in the distribution network such as energy shortages, unacceptable voltage fluctuations, transformer overloading, and energy losses.

Several research works have studied the EV charging scheduling problem in order to reduce the cost of it on customers and to reduce peak to average power ratio [1]. Authors in [2] proposed a management system for the charging of electrical vehicles so as to reduce the cost and maintain the loss to the minimum. The proposed scheme assigns preference according to the time zones with different energy tariffs to the customers for charging their 
vehicle. Authors in [3] simulated EV charging model for individual EV load profile under RTP to minimize electricity payment of users. In [4], the authors studied online peakminimizing algorithms for an aggregator, which solves the problem for handling large charging jobs and finish it on deadline. In [5] this shows V2G, G2V, V2H, different charging operation targeting different things. In [6] charging multiple storage medium at same time remains problems which are addressed using droop control. In [7] it shows the compensating the system on instantaneous and reactive power, it defines instantaneous power in the time domain. In [8] it shows about the battery management depending upon various factors like temperature etc. and how does it affect during charging. In [9] it shows that application of droop control while producing energy renewable sources, it uses it to improve voltage regulation. In [10] it shows charging of Li battery using dc link. It shows fast charging using (constant current/ constant voltage) stable and reliable characteristics are observed using it. In order to meet the owner requirement and the limit of the grid the design of the system is made to achieve both. Bidirectional power is the major requirement for the situation. So the aggregator plays an important role for it.

In this work, a charging algorithm for the $\mathrm{EV}$ is proposed. The priority determination rules are calculated based on the state of charge (SoC) of the battery. It is defined into three different priority levels as high, medium and low. The charging service will be allocated to EVs with higher priorities without violating the grid operational constraints. The proposed algorithm outperforms the conventional charging method. The entire paper is organized as follows: Segment 2 deals with the overall detailed system description. Segment 3 describes the priority charging algorithm and segment 4 details the results and the discussions on the same. Segment 5 outlines the conclusion of the entire work reported in the paper.

\section{SYSTEM OVERVIEW}

The overall system architecture is shown in Figure 1. It consists of a DC Micro grid, EVs, energy management system (EMS) and control system for load sharing and priority charging/discharging. The control system logic works on droop control. All the EVs are connected in parallel with the grid and the control logic is applied for charging and discharging. The control loop has the following functions:

- Get information from new arrival EVs

- Determine the soc

- Implement priority charging.

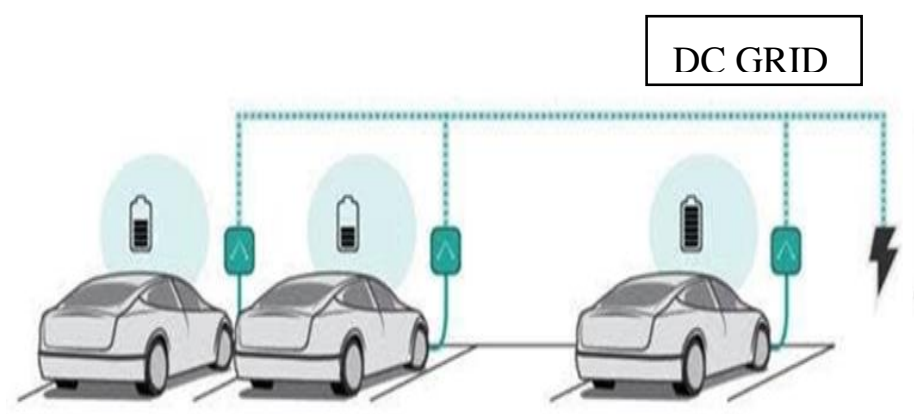

Figure 1 Overall system architecture 


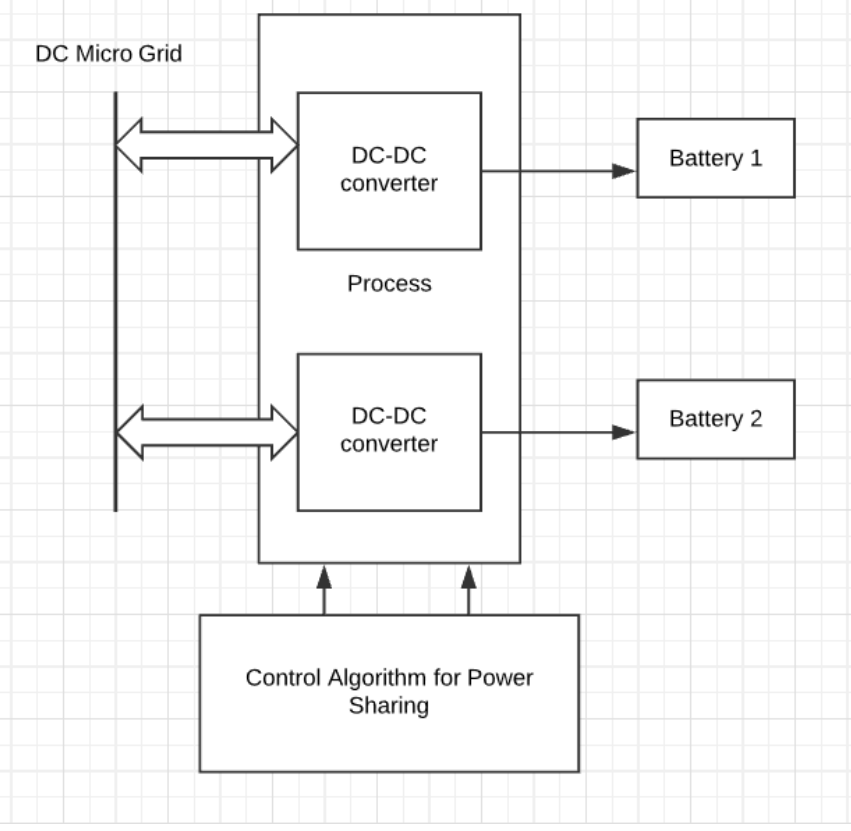

Figure 2 Basic Block Diagram

The overall block diagram is shown in fig 2 . The micro grids are connected to a dc-dc converter and are connected to batteries. The Power sharing is done using a control algorithm for the converters. The control algorithm for the rectifier helps it to adjust according to the need of the micro grid. It helps to save energy and increases efficiency of the model.

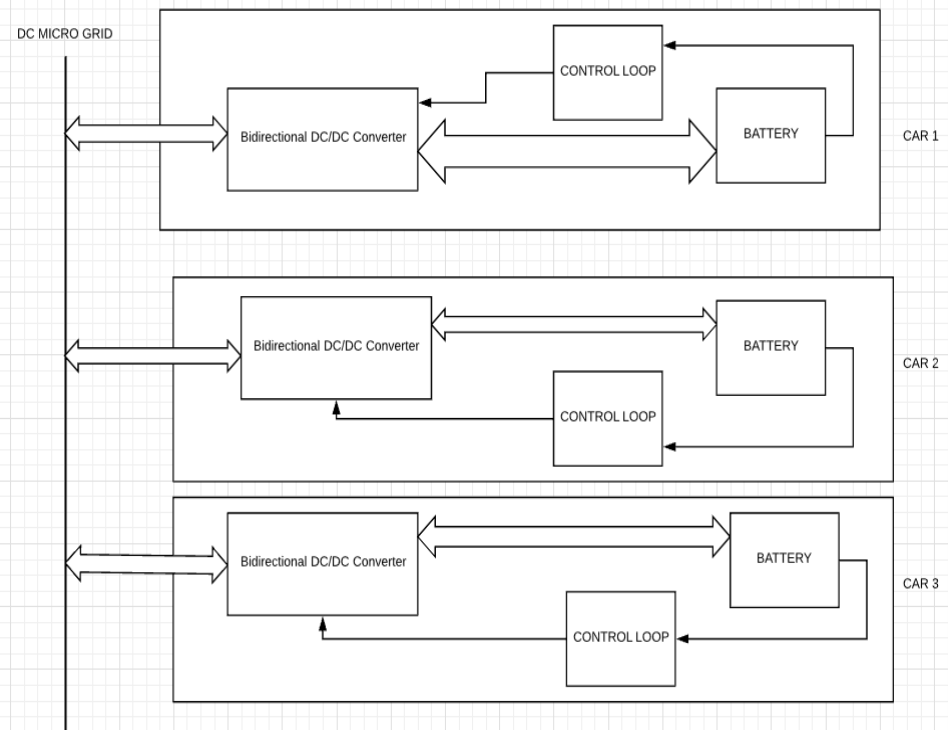

Figure 3 Diagram for control scheme

Fig 3 shows the block diagram for the power sharing in the micro grid. The batteries are connected to the micro grid through a bidirectional converter. The control loop is designed to control the converter connected to the battery. The control loop decides the amount of power required by each battery.

The block diagram explains the steps and process carried out in the system. The block diagram provides an insight on the working of the model and lays out a step by step working of the model. The block diagram for this model has been shown in figure 2 and figure 3 
We take the value of soc, current and voltage of two different EVs. The voltage is referred against a constant. We assume the standard voltage to be $48 \mathrm{v}$. The output of the comparator is multiplied with droop coefficient $(1 / \mathrm{K})$ and a reference current is obtained. The reference current is now compared with the source current. The output is given to a PI controller and the output is compared with a triangular wave.

The source is connected to the battery through a C-type dc-dc converter. The output pulses of the droop control are connected to the converter. This way the battery with the lower Soc gets the first priority and the load is shared.

\subsection{SoC Calculation}

SoC can be calculated in many ways. The state of charge $(\mathrm{SoC})$ is the available capacity as a percentage of a reference, most likely its current capacity. For this project soc has been calculated as the percentage of the released capacity relative to the battery rated capacity, given by the manufacturer (1).

'SOC $=$ Creleased $/$ Crated $\times 100 \%$

The equation of coulomb counting method is:

$$
\operatorname{SOC}(t)=\operatorname{SOC}(t-1)+\frac{I(t)}{Q_{n}} \Delta t .
$$

In general, the SOC of a battery is defined as the ratio of its current capacity $(\mathrm{Q}(\mathrm{t}))$ to the nominal capacity (Qn). The manufacturer gives the nominal capacity of the battery. The nominal capacity represents the total amount of charge that can be stored in each battery. The SOC can be defined as follows:

$$
\operatorname{SOC}(t)=\frac{Q(t)}{Q_{n}} \text {. }
$$

The various mathematical methods of estimation are classified according to methodology. The method of classification varies but in general there are following four categories.

(i) Direct measurement: The physical properties of the battery like voltage and impedance are used in this method.

(ii) Book-keeping estimation: In this method the reference is discharging current. The discharging current is integrated over time to find the soc value.

(iii) Adaptive systems: These systems are automatic and self-adjust the soc according to different condition and requirement. Many new adaptive systems can be developed.

(iv) Hybrid methods: these methods combine all the above described method and provides a optimal method of soc calculation.

The values of the components used in this model have been given in table 1 .

Table 1 System Parameters

\begin{tabular}{|l|l|}
\hline \multicolumn{1}{|c|}{ Components } & \multicolumn{1}{c|}{ Value } \\
\hline DC Grid Voltage & $48 \mathrm{v}$ \\
\hline Battery 1 & $24 \mathrm{v} / 5.4 \mathrm{Ah} / 20 \%$ \\
\hline Battery 2 & $24 \mathrm{v} / 5.4 \mathrm{Ah} / 40 \%$ \\
\hline RL Branch & $0.1 \mathrm{Ohms} / 1 \mathrm{e}-3 \mathrm{H}$ \\
\hline Inductance, L & $1 \mathrm{e}-3 \mathrm{H}$ \\
\hline
\end{tabular}




\section{PRIORITY DETERMINATION ALGORITHM}

In this work, the proposed optimal charging algorithm is based on assigning priority for EVs charging while considering the grid status. Figure 4 illustrates the flowchart for main charging algorithm. The main steps of the algorithm are as follows:

The initial soc, current and voltage value of the batteries are determined. The voltage is then compared using a comparator with a constant.

The output waveform is multiplied with the droop coefficient $(1 / \mathrm{K})$ and then the output is compared with the source current. The output is then tuned using a PI controller and the output is compared with a triangular waveform.

The output pulses obtained are used as pulses to trigger the thyristor of the converter and current to the battery is controlled. The battery with the lower soc draws more current and receives greater priority.

The rules for priority are based on the soc level of the batteries connected to the grid. After calculating EV priority, the control system assigns these priorities to EVs for charging, as given in Table 2 .

Table 2 Priority Determination

\begin{tabular}{|c|c|c|}
\hline Parameter & SOC \% & Voltage \\
\hline Priority & & \\
\hline HIGH & $\leq 20 \%$ & $\geq 30$ \\
\hline MEDIUM & $20<$ SOC $<80$ & $\leq 30$ \\
\hline LOW & $\geq 80 \%$ & $\leq 40$ \\
\hline
\end{tabular}

Three different priority levels high, medium and low are defined. With respect to the initial SOC of EV battery, it is defined a high priority for SOC less than $20 \%$, medium for $20-80 \%$ and low priority for SOC greater than $80 \%$.

The upper and lower limit has been selected after surveying the types of battery in the market and optimal working soc level of the batteries available in the market.

The charging will be enabled according to the priority set which will lead to the less constraint on the grid.

\section{RESULTS AND DISCUSSIONS}

In this section, the performances of the proposed control system for different cases are evaluated. A step by step approach has been used to develop this model. First a normal charging and discharging circuit has been simulated for a single battery. After designing a charging, a single battery, the model priority charging model was implemented and control system for the same was designed.

\subsection{Charging Process}

The result of fig 4 shows the charging of a single battery based on Soc level of the battery. According to the constraints if the soc $\leq 20 \%$ then the charging circuit is activated, and the battery is charged through the source.

From the waveform we can observe that the soc curve rises steadily and in a straight line which indicates the battery gets charged with stability and efficiency from $20 \%$ to $80 \%$ soc level. We can observe that for every $0.5 \mathrm{~s}$ the soc rises by $1 \%$ which shows fast charging. 


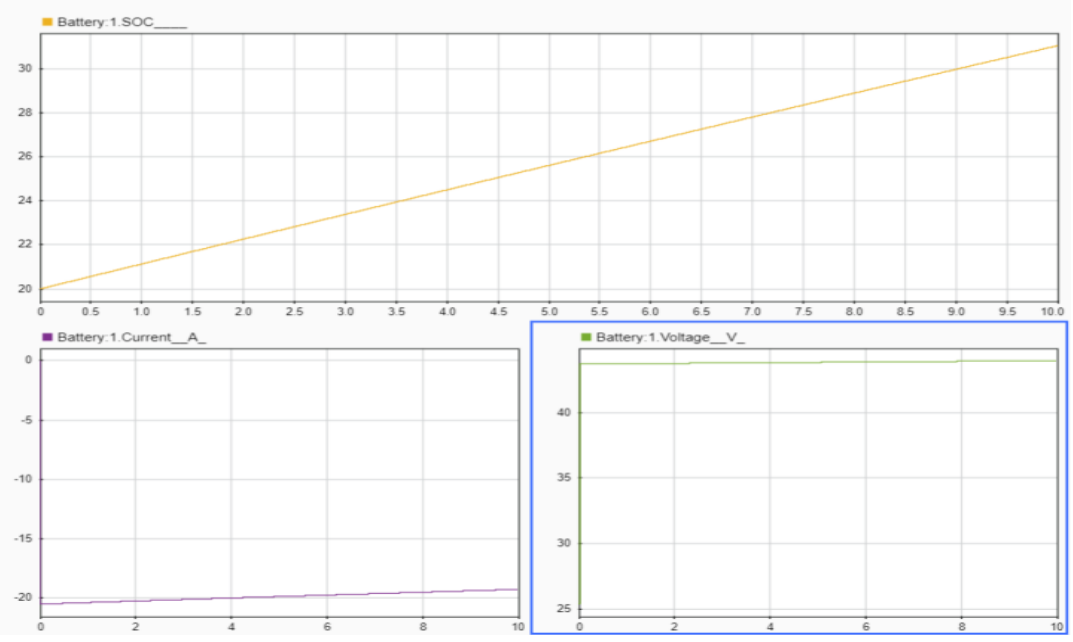

Figure 4 Charging of a Single Battery

\subsection{Discharging Process}

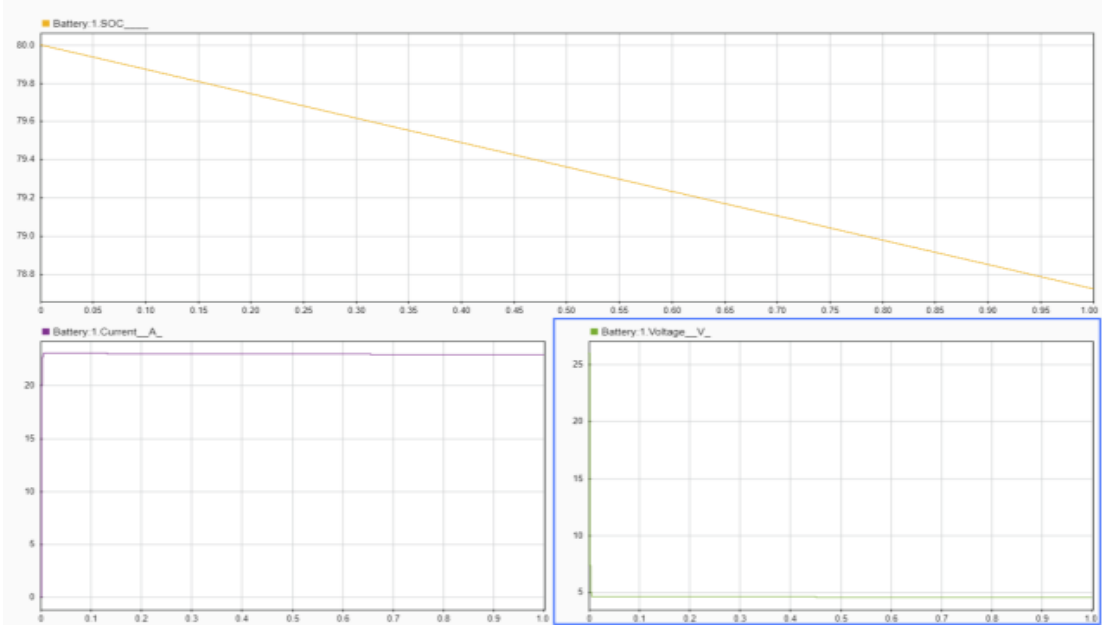

Figure 5 Discharging of a single battery

In this case the constraints are set that if Soc $\geq 80 \%$ then the battery discharged, and the power is supplied to the micro grid. As observed in figure the 6 the initial soc $\%$ of the battery is $80 \%$ and is slowly decreases to discharge the battery.

As observed from the graph the battery starts to discharge when soc is $80 \%$ and it steadily discharges through the discharging circuit. In this graph for every $0.15 \mathrm{~s}$ the soc level drops by $1 \%$. The battery is slowly discharged according to the limit and requirement of the grid.

\section{Case 1: Priority Charging}

The initial value of soc for battery 1 is $20 \%$ and battery 2 is $40 \%$. In fig 6 and 7 we can observe that the soc level increases and the batteries are charged.

Fig 8 displays power sharing in the source and amount of current drawn by each battery. It shows the way power is divided among the batteries and how the priority charging is implemented. 


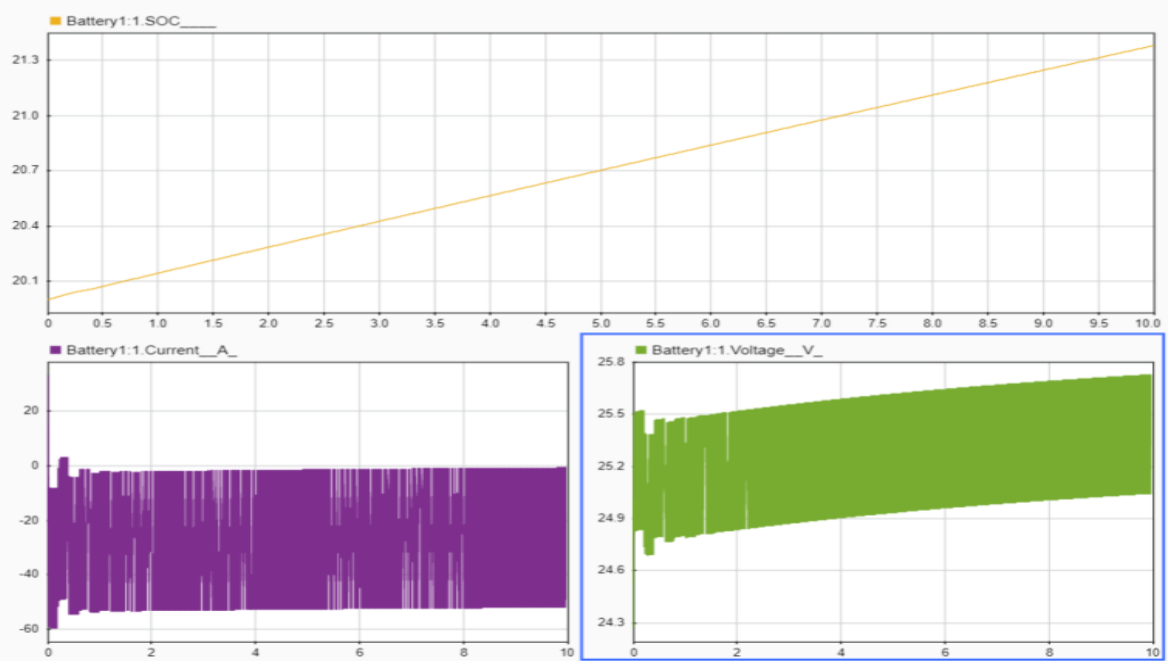

Figure 6 Characteristics of battery 1

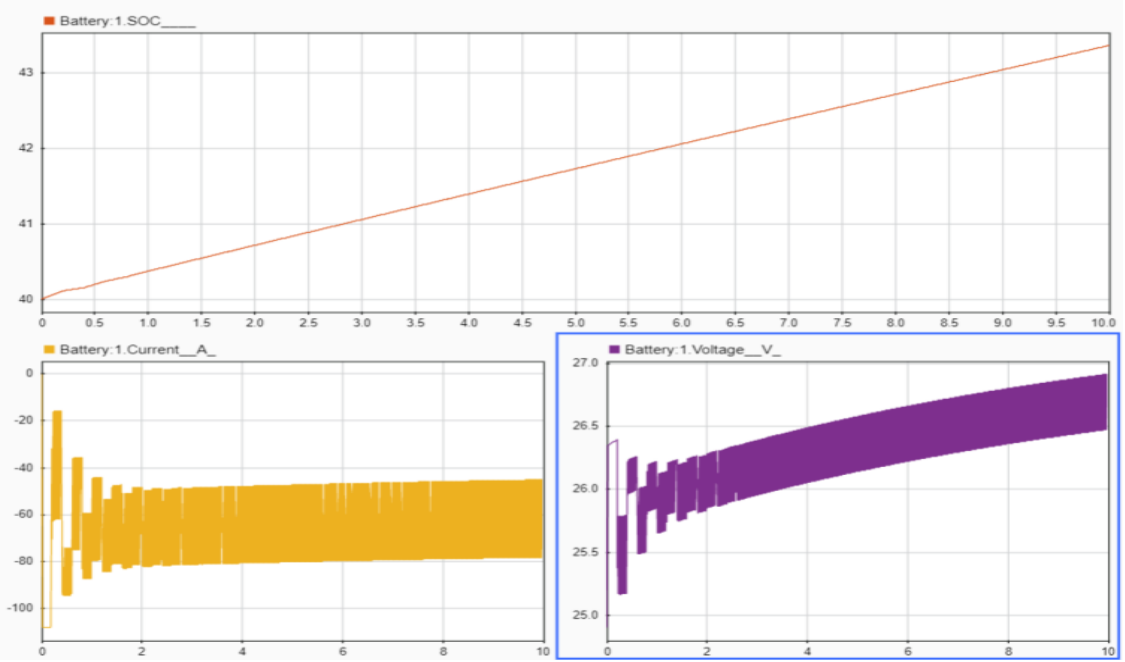

Figure 7 Characteristics of battery 2

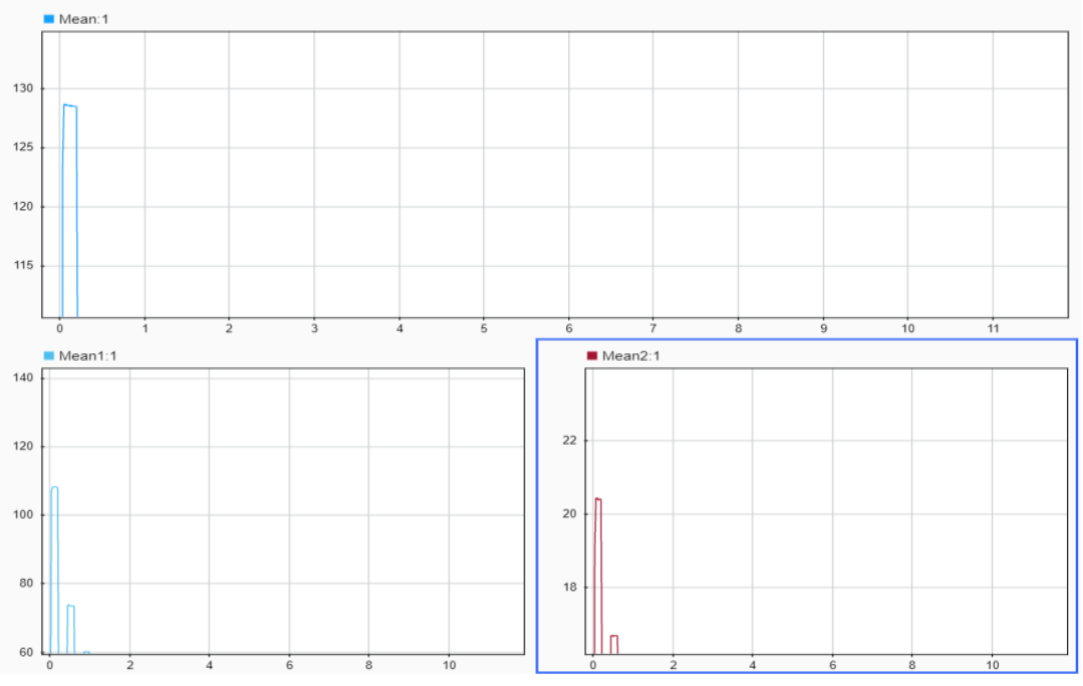

Figure 8 Current sharing among the batteries 
Figure 9 shows the result of priority charging. The source current is divided according to the priority. Battery 2 which has the more soc draws less current and battery 1 which has less 20 draws more current from the source.

The simulation result verifies that the control system developed is working efficiently and priority charging has been implemented. The proposed algorithm is working according to the simulation results obtained.

\section{CONCLUSION}

The working of EV charging connected to the dc micro grid through priority charging method is discussed in this work. This paper shows the charging number of the batteries connected to the grid. This paper shows some application of droop control for power distribution to the different batteries connected to the grid and power is to be sent the simultaneously. The paper shows the application of the priority charging which shows an efficient way of charging. The priority charging uses soc as the parameters for differentiating between different connected to it. This segregation is done by algorithm running for it which compares by soc. This makes a control loop which takes the feedback from the battery and give to the system for making the priority. Battery charges using voltage and current where one parameter is kept constant and other is increased or decreased based on the priority. The battery with less soc is given more priority. This method is applied in the simulation and results are shown in above section in the graph. The application of the project in hardware can be done by doing some changes in the simulation.

\section{REFERENCES}

[1] Q. QDR, (2006) "Benefits of demand response in electricity markets and recommendations for achieving them," U. S. Department of Energy.

[2] Z. Wang and R. Paranjape, (2015) "Optimal scheduling algorithm for charging electric vehicle in a residential sector under demand response," in 2015 IEEE Electrical Power and Energy Conference (EPEC), pp. 45-49.

[3] A. H. Mohsenian-Rad and A. Leon-Garcia, (2010) "Optimal Residential Load Control With Price Prediction in Real-Time Electricity Pricing Environments," IEEE Trans. Smart Grid, vol. 1, no. 2, pp. 120-133.

[4] S. Zhao, X. Lin, and M. Chen, (2013) "Peak-minimizing online EV charging," in 2013 51 st Annual Allerton Conference on Communication, Control, and Computing (Allerton), 2013,pp. 46-53,

[5] Bidirectional Battery charger with Grid-vehicle, Vehicle-Grid, Vehicle to home Technologies (2013) (J. G. Pinto, Vítor Monteiro, Henrique Gonçalves, Bruno Exposto, Delfim Pedrosa, Carlos Couto, João L. Afonso)IEEE ICON November, pp. 5934-5939

[6] Droop Controller Design Methods for Isolated DC- DC Converter in DC Grid Battery Energy Storage system Application (2013) (Yu Du, Alex Q. Huang, Xunwei Yu, Jun li) IEEE APEC.pp 1630-1637

[7] Instantaneous p-q Power Theory for Compensating Non sinusoidal Systems (2008) (E. H. Watanabe, H. Akagi, and M. Aredes). 2008 International School on Nonsinusoidal Currents and Compensation, Lagow, pp. 1-10

[8] Research on battery charging strategy with charging and temperature control awareness (2018) (Min Ye, Haoran Gong, Rui Xiong and Hao Mu) IEEE 2018.vol. 6, pp. 6419364201 
[9] Droop Control Method for Parallel DC Converters used in Standalone PV-Wind Power Generating System (2015) (Reshma Mary Thomas, Deepu Jose) EIJRET. Vol. 6, pp 297302

[10] Study on Li-Battery Charge Control based on DC- Grid (2011) (Youngin Lee, D.H Han, C.H Ban, Gyuha Choe, J.M Eun, Bayasgalan D) 8th International Conference on Power Electronics - ECCE Asia , pp 2241-2247.

[11] G. Mahesh Kumar, Y. Damodharam, Velappagari Sekhar, S. Suresh, (2014) Integration of Renewable Resources for DC Micro Grid Applications, International Journal of Electrical Engineering and Technology, 5(8), pp. 1-12 